\title{
Oblique launching of optical surface waves by a subwavelength slit
}

\author{
A. Yu. Nikitin, ${ }^{1,2, *}$ F. J. García-Vidal, ${ }^{3}$ and L. Martín-Moreno ${ }^{1, \dagger}$ \\ ${ }^{1}$ Instituto de Ciencia de Materiales de Aragón and Departamento de Física de la Materia Condensada, CSIC-Universidad de Zaragoza, \\ ES-50009, Zaragoza, Spain \\ ${ }^{2}$ A.Ya. Usikov Institute for Radiophysics and Electronics, Ukrainian Academy of Sciences, 12 Acadamician Proskura Street, \\ UA-61085 Kharkov, Ukraine \\ ${ }^{3}$ Departamento de Física Teórica de la Materia Condensada, Universidad Autónoma de Madrid, ES-28049 Madrid, Spain
}

(Received 4 February 2011; revised manuscript received 22 March 2011; published 26 April 2011)

\begin{abstract}
The electromagnetic field on the metal surface launched by a subwavelength slit is analytically studied for the case when the fundamental mode inside the slit has a wave-vector component along the slit axis (conical mount). Both near-field and far-field regions are discussed, and the role of surface plasmon-polaritons and Norton waves is revealed. It is shown that the distance from the slit at which Norton waves are more intense than surface plasmons decreases with the parallel wave vector. Additionally, it is found that the $s$-polarization component, while present for any nonzero parallel wave vector, only weakly contributes to the Norton waves.
\end{abstract}

DOI: 10.1103/PhysRevB.83.155448

PACS number(s): 42.25.Bs, 41.20.Jb, 42.79.Ag, 78.66.Bz

\section{INTRODUCTION}

Launching surface plasmon-polaritons (SPPs) along metal surfaces has recently attracted a lot of interest for its possible application in integrated optical devices. ${ }^{1-4}$ One of the most common configurations used employs systems with translational symmetry in one direction, as a subwavelength slit ${ }^{5,6}$ or a line defect. ${ }^{7,8}$

Very recently, several studies have been devoted to finding simple analytical models for the electromagnetic (EM) field radiated by a single slit, which provide insight into the relevant physical processes. ${ }^{9-13}$ It has been found that the field at the surface presents a rich behavior as a function of both frequency and distance to the slit. Typically, at distances less that 2-3 wavelengths, the field presents a complex spatial dependence ${ }^{14}$ (which is sometimes phenomenologically described as composed of a SPP plus a "creeping" or "quasicylindrical" wave, $\left.\mathrm{CW}^{5,13}\right)$. At larger distances, there is an intermediate regime where the EM field is dominated by the SPP contribution, and a long-distance regime where the SPP has become negligible and the field is that of a Norton wave (NW). Recall that NWs always appear at the absorbing metal surfaces under local excitation; they show algebraic decay both along the metal surface and in the perpendicular direction, and oscillate with the period of free-space wavelength. All the previously cited works focused on the case of field propagation perpendicular to the slit, and they did not address the situation of non-normal incidence of light into the defect. This is a serious deficiency, because SPP scattering effects are easier to detect when the SPP is launched obliquely, i.e., when its wave vector has a component along the slit axis. ${ }^{7,8,15-18}$

In this paper we fill this gap, presenting an analysis of the EM fields on the metal surface obliquely launched by a subwavelength one-dimensional aperture. Such a launching could be realized by illuminating a subwavelength slit in a thick metal film by a plane wave in the conical mount (see Fig. 1). Both $x$ and $y$ components of the incident wave vector can be nonzero. Since the problem possesses a translational symmetry in the $y$ direction, only the $y$ component of the wave vector is conserved, and since the slit is supposed to be thin, the $x$ component inside the slit is imposed by the fundamental capacitor-like mode: $k_{x}=0$. For this reason, the $x$ component of the incident wave vector is not important to our problem. However, there are some restrictions for the polarization of the incident wave: the electric field must have a component perpendicular to the slit, i.e., $E_{i x} \neq 0$, in order for the fundamental mode to be excited (all other modes inside the slit being exponentially suppressed) and the energy to be transferred to the outgoing face of the film. From now on, we will denote this configuration as "oblique incidence." We would like to stress that due to $k_{y} \neq 0$ the field emerging from the outgoing aperture gains both $p$ - and $s$-polarization components. Here we do not consider the part of the problem related to the transmission and reflection efficiencies, but concentrate on the electric field pattern on the outgoing face of the film. We thus only need to know the Green's function of our system and the field at the outgoing face of the slit. For this we apply the mathematical methods described in Ref. 20, which have been previously used for obtaining the field at the metal surface radiated both by a single slit for $k_{y}=0$ (Refs. 9,11, and 12) and by a subwavelength hole. ${ }^{19}$

Anticipating things, we would like to stress that the situation where $k_{y} \neq 0$ presents two main differences with the case $k_{y}=0$ : (i) the transmitted electric fields have components parallel to the slit axis and (ii) the decay length of the excited SPP in the direction across the slit diminishes. The latter fact favors bringing closer to the slit the region where Norton waves dominate.

\section{ANALYTICAL SOLUTION}

Let us consider a plane monochromatic wave incident onto a thick metallic film with a subwavelength slit centered at $X=0$. The wave vector of the incident wave is $\mathbf{k}_{i}$, forming an arbitrary angle with respect to the $O z$ and $O y$ axes, and the wave is arbitrarily polarized (see Fig. 1), but $E_{i x} \neq 0$. We consider the symmetric configuration, in which the dielectric permittivity of both substrate and superstrate is $\varepsilon$, while the dielectric constant inside the slit is arbitrary. Throughout the paper, distances in lower case letters are expressed in dimensionless units, defined as $x=k_{\omega} X, z=k_{\omega} Z$, and the dimensionless wave-vector components are denoted as $q_{x, y}=k_{x, y} / k_{\omega}$, where $k_{\omega}=2 \pi / \lambda$ 


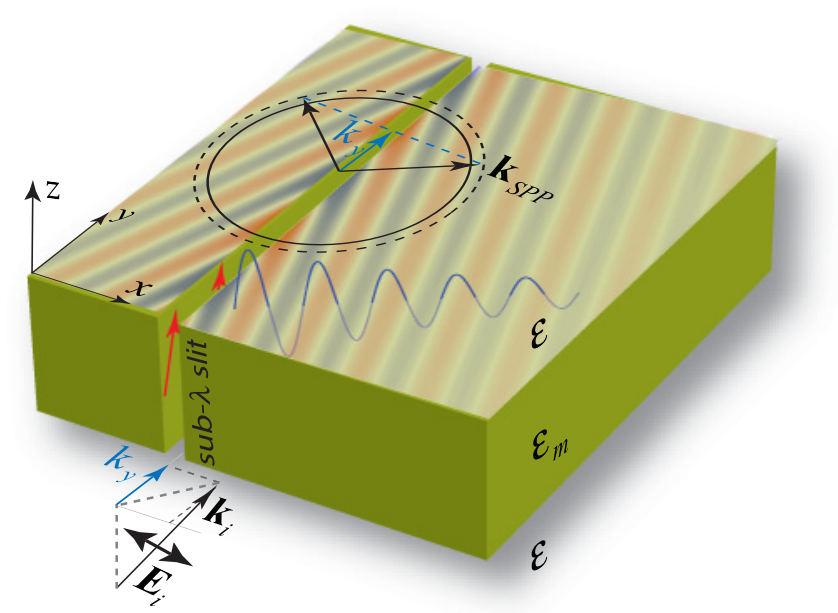

FIG. 1. (Color online) Geometry of the studied system. A plane electromagnetic wave impinges onto a subwavelength slit, placed in an optically thick film. The angle of incidence is arbitrary provided the electric field points perpendicular to the slit axis. The slit aperture at the transmission region generates a field at the surfaces, propagating nonperpendicularly to the slit axis. The solid circle represents a slice of the light cone for a fixed frequency, while the dashed one is the slice of the "SPP cone." Arrows inside the slit indicate the propagation direction of the fundamental mode.

(so that, for instance, the light cone in vacuum corresponds to $q=1)$.

\section{A. General analytical expression for the field}

Starting from the Lippmann-Schwinger integral equation and taking into account that the field in the slit points along the $x$ direction, then $\mathbf{E}(x, y, z) \simeq \mathbf{e}_{x} E_{x}(x, z) e^{i q_{y} y}$, the expression for the transmitted field, simplifies to (see Appendix for details)

$$
\mathbf{E}(x, z)=C \int_{L} d x^{\prime} \mathbf{G}\left(x-x^{\prime}, z\right) E_{x}\left(x^{\prime}, z=-\delta\right),
$$

where $C=i \sqrt{\varepsilon_{m}-\varepsilon} / k_{\omega}$. The dielectric constants of the dielectric in the outgoing region and the metal are $\varepsilon$ and $\varepsilon_{m}$, respectively, and $\delta$ is the skin depth in the metal. The integration in $x^{\prime}$ is performed across the slit area. The cyclic dependency upon the coordinate $y, \propto e^{i q_{y} y}$ is omitted here and in what follows. $\mathbf{G}(x, z)$ is the $x$ column of Green's dyadic, $\mathbf{G} \equiv \hat{G} \mathbf{e}_{x}$ (with $\mathbf{e}_{x}$ being a unitary vector along the $O x$ axis), whose angular representation for the case of arbitrary $k_{y}$ reads

$$
\begin{aligned}
& \mathbf{G}^{p}(x, z)=\frac{i k_{\omega}}{4 \pi} \int \frac{d q_{x}}{q^{2} \sqrt{\varepsilon_{m} \varepsilon}} t^{p}\left(\begin{array}{c}
q_{x}^{2} q_{z} \\
q_{x} q_{y} q_{z} \\
-q_{x} q^{2}
\end{array}\right) e^{i\left(q_{x} x+q_{z} z\right)}, \\
& \mathbf{G}^{s}(x, z)=\frac{i k_{\omega}}{4 \pi} \int \frac{d q_{x}}{q^{2} q_{z m}} t^{s}\left(\begin{array}{c}
q_{y}^{2} \\
-q_{x} q_{y} \\
0
\end{array}\right) e^{i\left(q_{x} x+q_{z} z\right)},
\end{aligned}
$$

where $\mathbf{G}=\mathbf{G}^{p}+\mathbf{G}^{s}$, indices $p$ and $s$ stand for the corresponding polarizations, and $t_{p}$ and $t_{s}$ are Fresnel transmission coefficients for the metal-dielectric interface, given by

$$
t^{s}=\frac{2 q_{z m}}{q_{z m}+q_{z}}, \quad t^{p}=\sqrt{\frac{\varepsilon_{m}}{\varepsilon}} \frac{2 q_{z m} \varepsilon}{q_{z} \varepsilon_{m}+q_{z m} \varepsilon},
$$

with $q^{2}=q_{x}^{2}+q_{y}^{2}, q_{z}=\sqrt{\varepsilon-q^{2}}$, and $q_{z m}=\sqrt{\varepsilon_{m}-q^{2}}$. The branches of $q_{z}, q_{z m}$ must be chosen in accordance with the radiation conditions $\operatorname{Im}\left(q_{z}, q_{z m}\right) \geqslant 0$. It should be noted that for $q_{y}=0$ the integrals transform to the case of in-plane launching (see Refs. 11 and 12), and the $s$ components of the fields vanish.

For narrow (subwavelength) slits, the field inside the slit can be taken as independent of $x$ and, therefore, the outgoing slit aperture is equivalent to the effective two-dimensional electric dipole located on the metal surface: ${ }^{11} \mathbf{E}(x, z)=\mathbf{G}(x, z) p^{\text {eff }}$, where $p^{\text {eff }}=a C E_{x}(0, z=-\delta)$ and $a$ is the width of the slit (in dimensionless units).

The numerical computation of the integrals in Eq. (2) are notoriously difficult, due to the simultaneous presence of poles, branch cuts, and strongly oscillatory factors. Using a special mathematical treatment based on the steepest descent method, ${ }^{20}$ an accurate analytical representation of the asymptotic behavior of the field is possible. We present here the final result for the field at the metal surface $z=0$; the mathematical details can be found in the Appendix. Introducing the following notation

$$
\mathbf{G}(x, z=0)=\frac{i k_{\omega}}{2 \pi} \mathbf{g}(x),
$$

we have in the region $q_{0} x \gg 1$,

$$
\begin{array}{r}
\mathbf{g}(x) \approx i \pi \mathbf{C}_{p} e^{i q_{x p} x} \operatorname{erfc}\left(-i s_{p} \sqrt{q_{0} x}\right)+e^{i q_{0} x} \sqrt{\frac{\pi}{q_{0} x}} \frac{\mathbf{C}_{p}}{s_{p}} \\
+\frac{\sqrt{\pi} e^{i q_{0} x}}{4 q_{0} x \sqrt{q_{0} x}}\left[\frac{2 \mathbf{C}_{p}}{s_{p}^{3}}+2 \sqrt{2} e^{-i \frac{3 \pi}{4}}\left(\mathbf{f}^{s}+\mathbf{f}^{p}\right)\right] .
\end{array}
$$

In this equation $q_{0}=\sqrt{\varepsilon-q_{y}^{2}}$ presents the inverse spatial period along the $x$ axis of the algebraically decaying terms, $q_{p}=$ $\sqrt{\varepsilon \varepsilon_{m} /\left(\varepsilon+\varepsilon_{m}\right)}$ is the modulus of the in-plane component of the SPP momentum, and $q_{x p}=\sqrt{q_{p}^{2}-q_{y}^{2}}$ is its $x$ component. The factor $s_{p}=e^{-i \pi / 4} \sqrt{q_{x p} / q_{0}-1}$, appearing in the argument of the complementary error function, erfc, is the position of the pole in the complex plane where the steepest-descent integration is made (see Appendix). It has an important significance, being responsible for the asymptotic expansion of the error function, whose argument is the the square root of the socalled numerical distance introduced by Sommerfeld. Another property of $s_{p}$ is that $\left|s_{p}\right|^{2}$ quantify the distance in complex $q$ space between the SPP pole and the branch-point placed at $q=\sqrt{\varepsilon}$ (i.e., at $q_{z}=0$ ).

The terms in Eq. (5) containing $\mathbf{C}_{p}$ come from the singular part of the integrals in Eq. (2) appearing for $p$ polarization. $\mathbf{C}_{p}$ are the residues at $q_{x}=q_{p x}$ :

$$
\mathbf{C}_{p}=\frac{\varepsilon_{m} q_{z p} \sqrt{\varepsilon_{m}-\varepsilon}}{q_{p}^{2}\left(\varepsilon^{2}-\varepsilon_{m}^{2}\right)}\left(\begin{array}{c}
q_{x p} q_{z p} \\
q_{y} q_{z p} \\
-q_{p}^{2}
\end{array}\right)
$$

where $q_{z p}=\varepsilon / \sqrt{\varepsilon+\varepsilon_{m}}$.

Finally, $\mathbf{f}^{s, p}$ are contributions to the algebraically decaying term, which in this one-dimensional geometry goes as 


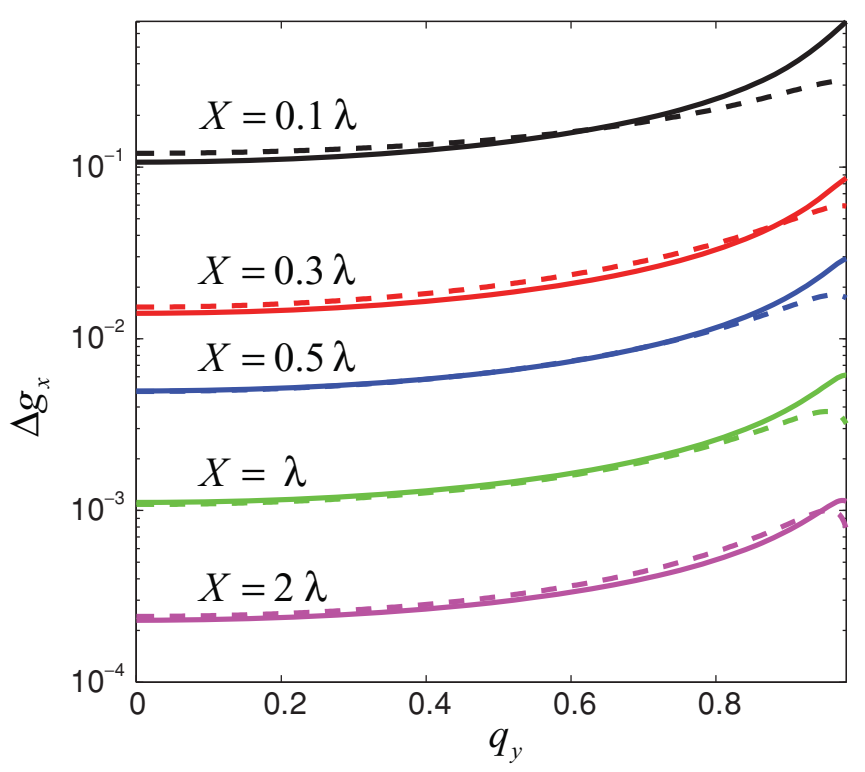

FIG. 2. (Color online) Relative error $\Delta g_{x}$ as a function of $q_{y}$ in the case of a gold surface. Continuous curves correspond to $\lambda=$ $700 \mathrm{~nm}$, while discontinuous ones are for $\lambda=540 \mathrm{~nm}$.

$\sim 1 / x^{3 / 2}$, which dominate the far-field region at the surface (unless the system is completely absorptionless)

$$
\begin{gathered}
\mathbf{f}^{s}=\frac{2 q_{y} q_{0}^{2}}{\varepsilon\left(\varepsilon_{m}-\varepsilon\right)}\left(\begin{array}{c}
-q_{y} \\
q_{0} \\
0
\end{array}\right), \\
\mathbf{f}^{p}=\frac{2 q_{0}^{3}}{\varepsilon^{2}}\left(\begin{array}{c}
q_{0} \\
\frac{q_{y}}{\sqrt{\varepsilon_{m}-\varepsilon}}
\end{array}\right) .
\end{gathered}
$$

It is remarkable that the region for validity of the solution (4)-(7) is much less restrictive than $q_{0} x \gg 1$ (i.e., $x \gg$ $1 / \sqrt{\varepsilon-q_{y}^{2}}$ ), similar to what occurred in the case of $q_{y}=0 .{ }^{11}$ Notice, nevertheless, that as $q_{y}$ increases, this formal condition is fulfilled for larger values of $x$, which explains that, at a fixed distance, the relative error in the field increases with $q_{y}$. To characterize the relative error, we have introduced the following function $\Delta g_{\alpha}=\left|\left(g_{\alpha}-g_{\alpha}^{\text {num }}\right) / g_{\alpha}^{\text {num }}\right|$ with $\alpha=x, y, z$, where $g_{\alpha}^{\text {num }}$ correspond to precise numeric calculations and $g_{\alpha}$ is given by Eqs. (5)-(7). Except for $q_{y} \approx 1$ this error is not very sensitive to the value of $\varepsilon_{m}$, and, therefore, almost independent of wavelength (from the optical region to longer wavelengths). We have checked that the relative error does not exceed a few percent for distances as small as $X \sim \lambda / 10$, and already for $X$ of order of a wavelength the error has reduced down to $0.1 \%$ at $q_{y}=0$ (and $\sim 0.5 \%$ at $q_{y} \approx 1$ ), see Fig. 2 . Here and below we take the dielectric permittivity of the metal, $\varepsilon_{m}$, from Ref. 21.

\section{B. Perfect electric conductor limit}

Before analyzing the case of a real conductor, let us consider the limiting case of a perfect electric conductor (PEC), characterized by $\varepsilon_{m} \rightarrow-\infty$. Then the electric field at the surface is perpendicular to it and, for a very thin slit, can be analytically calculated using directly Eqs. (1) and (2):

$$
\mathbf{E}^{\mathrm{PEC}}(x, 0)=-\mathbf{e}_{z} \frac{q_{0}}{2} a H_{1}^{(1)}\left(q_{0}|x|\right) E_{x}^{\mathrm{PEC}}(0,0),
$$

with $H_{1}^{(1)}$ being the Hankel function of first order.

The PEC limit of the asymptotic expansion has to be taken with care, as Green's dyadic tends to zero as $\mathbf{G} \sim 1 / \sqrt{\varepsilon_{m}}$. This is natural, since the dipole placed on the metal interface and oriented along it cannot radiate due to cancellation of the field by the image dipole. However, the effective dipole of the slit diverges as $p^{\text {eff }} \sim \sqrt{\varepsilon_{m}}$, so that the product $\mathbf{G} p^{\text {eff }}$ remains finite. If we substitute Eqs. (4)-(7) into Eq. (1) and perform the PEC limit, we arrive at the asymptotic expansion for Eq. (8). This expansion consists of just one term, where the Hankel function is replaced by its asymptotic term $H_{1}^{(1)}\left(q_{0}|x|\right)=$ $-\sqrt{2 i /\left(\pi q_{0} x\right)} e^{i q_{0} x}$. Thus, our asymptotic expansion recovers the PEC result, up to terms of order $O\left(x^{-5 / 2}\right)$.

\section{Far-field asymptotic}

For long distances or, more precisely, when $x q_{0}\left|s_{p}\right| \gg 1$, we can obtain a simplified expression from Eq. (5) [exact up to $\left.O\left(x^{-5 / 2}\right)\right]$ :

$$
\mathbf{g}(x)=\mathbf{g}_{\mathrm{SPP}}(x)+\mathbf{g}_{\mathrm{NW}}(x)
$$

where $\mathbf{g}_{\mathrm{SPP}}$ is the contribution from the SPP pole (arising from the first term in the asymptotic expansion of the complementary error function)

$$
\mathbf{g}_{\mathrm{SPP}}(x)=2 \pi i \mathbf{C}_{p} e^{i q_{x p} x},
$$

and $\mathbf{g}_{\mathrm{NW}}(x)=\mathbf{g}_{\mathrm{NW}}^{p}(x)+\mathbf{g}_{\mathrm{NW}}^{s}(x)$ is an algebraically decaying term, with contribution from both polarizations

$$
\mathbf{g}_{\mathrm{NW}}^{\sigma}(x)=\frac{\sqrt{\pi} e^{-i \frac{3 \pi}{4}}}{q_{0} \sqrt{2 q_{0}}} \mathbf{f}^{\sigma} \frac{e^{i q_{0} x}}{x \sqrt{x}}, \quad \sigma=p, s .
$$

This field component presents the two-dimensional analog of the Norton waves (NW) discovered theoretically almost a century ago by Norton, ${ }^{22}$ when analyzing the radiation of radio waves by a point dipole placed over the Earth's surface (represented by a lossy dielectric).

We would like to stress that the approximation (9)-(11) is not applicable for PECs since, in this case, $s_{p}=0$ and the condition for validity of the asymptotic expansion is not fulfilled for any distance.

In the remaining part of the article we analyze the dependency of the fields upon $q_{y}$. We concentrate on the case of a vacuum-gold interface setting $\varepsilon=1, \varepsilon_{m}=\varepsilon_{A u}$. The effects generated by changing $\varepsilon$ were considered in Ref. 12 .

\section{ILLUSTRATIVE RESULTS AND THEIR DISCUSSION}

Before presenting the dependence of the field pattern on $q_{y}$, for completeness and in order to make the comparative analysis easier, we briefly review the case $q_{y}=0$ (extensively studied in Refs. 10-13). Figure 3 shows a representative case ( $\lambda=540 \mathrm{~nm}$ and $q_{y}=0$ ) for the dependence on distance to the slit of the electric field at the metal surface. The fields for both vacuum-gold and vacuum-PEC interfaces are shown, under the assumption that they are launched by slits with the 


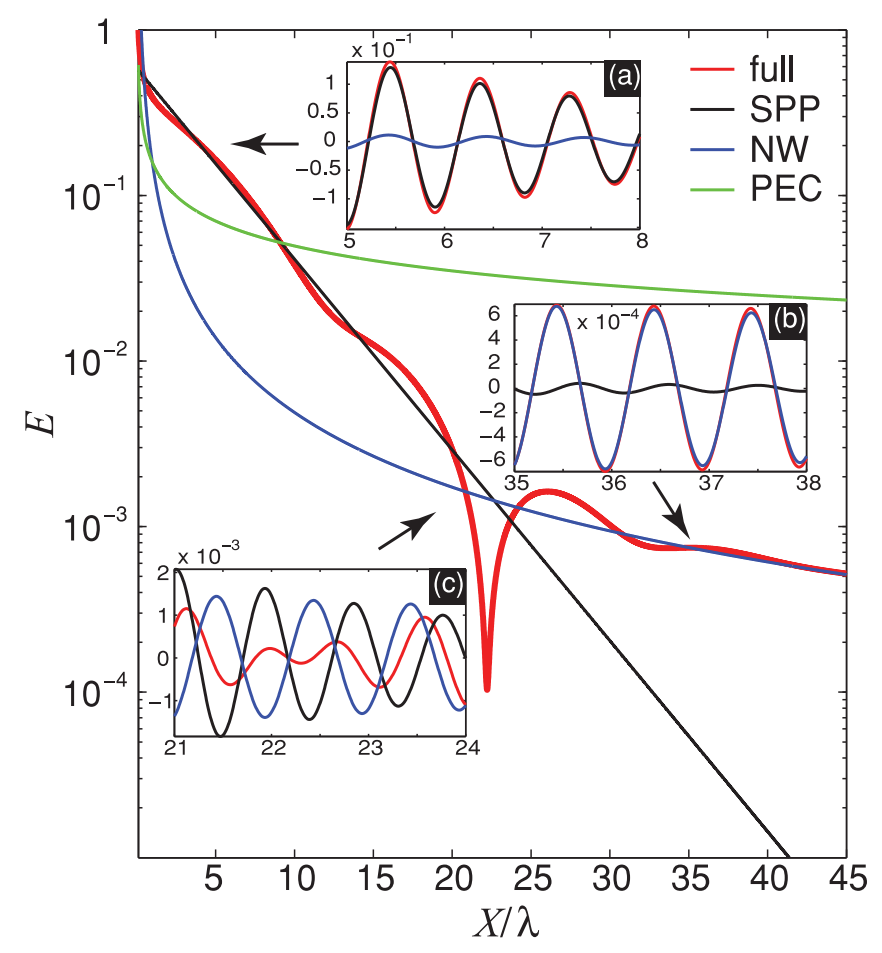

FIG. 3. (Color online) Dependency upon distance from the slit of the electric field modulus at the gold surface. The wavelength is $\lambda=540 \mathrm{~nm}$ and $q_{y}=0$. Together with the total field, both SPP and NW contributions, and the PEC case are also plotted. The insets show the real part of the $z$ component of the total, SPP, and NW fields, in different spatial regions. All fields are normalized to $E(X=$ $0.1 \lambda, z=0)$, which is taken as a representative value in the near field.

same amplitude of the electric field on their exit apertures. The spatial dependence of the field is quite different in these two cases. Instead of the cylindrical wave-type algebraic decay $\propto 1 / \sqrt{x}$ along the PEC surface, the field along the vacuum-gold interface sufficiently away from the slit shows two different behaviors: exponentially decaying SPP and algebraically decaying NW. As a reference for the boundary between these two regions, let us introduce crossover distance, $X_{c}$, as the distance where the amplitude of the SPP and NW are equal. It must be stressed that the chosen wavelength, $\lambda=540$ $\mathrm{nm}$, and the type of metal (gold) does not represent a special case with some particular properties, but corresponds to a small value of $X_{c}$. Otherwise, the physics for this case is as rich as for other wavelengths.

As shown in Fig. 3, in a very close vicinity to the slit $x \ll 1$, the behavior of the field is complex and contains the contribution from all the angular spectrum of Green's function, or in other words, from all the density of EM states. Phenomenologically, the field in this region has been represented by a SPP plus an additional contribution (defined as the total field minus the SPP one) denoted either "creeping wave" or "quasicylindrical wave" (CW, see Refs. 5 and 13). As mentioned before, Eq. (5) faithfully represents the field (and thus the $\mathrm{CW}$ ) for $X \geqslant 0.1 \lambda$. As $X$ increases, all the smooth parts in the angular spectra in the integral are progressively canceled out due to integration with the oscillatory factor $\sim \exp \left(i q_{x} x\right)$, so that only the sharp regions (with width $\Delta q \sim 1 / x)$ of the spectrum give a finite net contribution. These regions correspond to the vicinities of either the pole $q=q_{p}$ (which is a feature of a finite width) or the kink $q=1$ (which has zero width in $q$ space). The field in the region where these two contributions dominate can be found by asymptotically expanding Eq. (5), see Sec. II C.

The electric fields corresponding to the SPP and NW terms are rendered in Fig. 3. At distances from the slit of order of one wavelength, both field amplitude and phase [see inset (a) in Fig. 3] are well approximated by the SPP contribution, and the influence of the NW is weak. The field is locally enhanced compared to the PEC case, and the efficiency of the SPP excitation depends upon wavelength. In the region close to the asymptote in the SPP dispersion relation $\left(\varepsilon_{m} \simeq-1\right)$, the density of electromagnetic states increases and so does the local field enhancement. However, while the mode becomes both slower and more confined, due to the increase of its wave vector, the absorption increases as well and the SPP mode is quenched at a smaller distance from the source.

At distances large enough so that, due to the absorption, the SPP is sufficiently damped, the contributions from SPP and NW are comparable, see inset (b) in Fig. 3. This typically occurs at $X_{c} \sim 6-9 L_{x}$, with $L_{x}=\lambda /\left[2 \pi \operatorname{Im}\left(q_{x p}\right)\right]$ being the SPP decay length along the $x$ axis. In the vicinity of $X_{c}$, the SPP and NW fields have similar amplitudes, so the modulus of their sum presents an interference behavior, leading to a set of maxima and minima. In the vicinity of the minima the energy flux is suppressed. In the dielectric media, for small values of $z$ the energy flux presents the interference between the SPP and cylindrical wave (appearing as the ray optics approximation) contributions; the Norton wave does not contribute to the energy flux since it decays faster that the cylindrical wave. Due to the energy conservation, the lines of the Poynting vector round the minima in the vicinity of the point $\left(X=X_{c}, Z=0\right)$ compensating the energy flux through the cylindrical wave.

Notice that in the optical region the SPP wave vector is close to the light cone, thus close to the NW one. However, $q_{p}$ largely increases close frequencies such that $\varepsilon_{m} \simeq-1$ (which, for good metals, occur at $\omega_{p} / \sqrt{2}$, where $\omega_{p}$ is the plasma frequency), in which case the total field given by Eq. (9) presents a fanciful two-scaled oscillatory behavior.

The third region is located beyond $X_{c}$, where the contribution from the SPP field is negligible [see inset (c) in Fig. 3]. The field then reaches its asymptotic behavior, which is given by the NW, oscillating with the spatial period given by the free-space wavelength and decaying algebraically as $\sim 1 / x^{3 / 2}$.

Let us now analyze the dependency of the fields upon the $y$ component of the wave vector. Figure (4) shows the spatial dependencies of the fields in the direction perpendicular to the slit for different $q_{y}$. There are two tendencies with the increase of $q_{y}$ : the amplitude of the field decreases, and the crossover distance $X_{c}$ diminishes. To explain this behavior, we represent $X_{c}$ as a function of $q_{y}$ [inset (a) to Fig. 4]. As can be seen from the curves where $X_{c}$ is normalized to the constant ( $q$-independent) value $L_{x}\left(q_{y}=0\right)$, the distance after which the NW dominates decreases as $q_{y}$ increases. However, the value of $X_{c}\left(q_{y}\right) / L_{x}\left(q_{y}\right)$ increases as $q_{y}$ increases, meaning that the crossover occurs at smaller absolute distances, but at larger decay lengths, specially close 


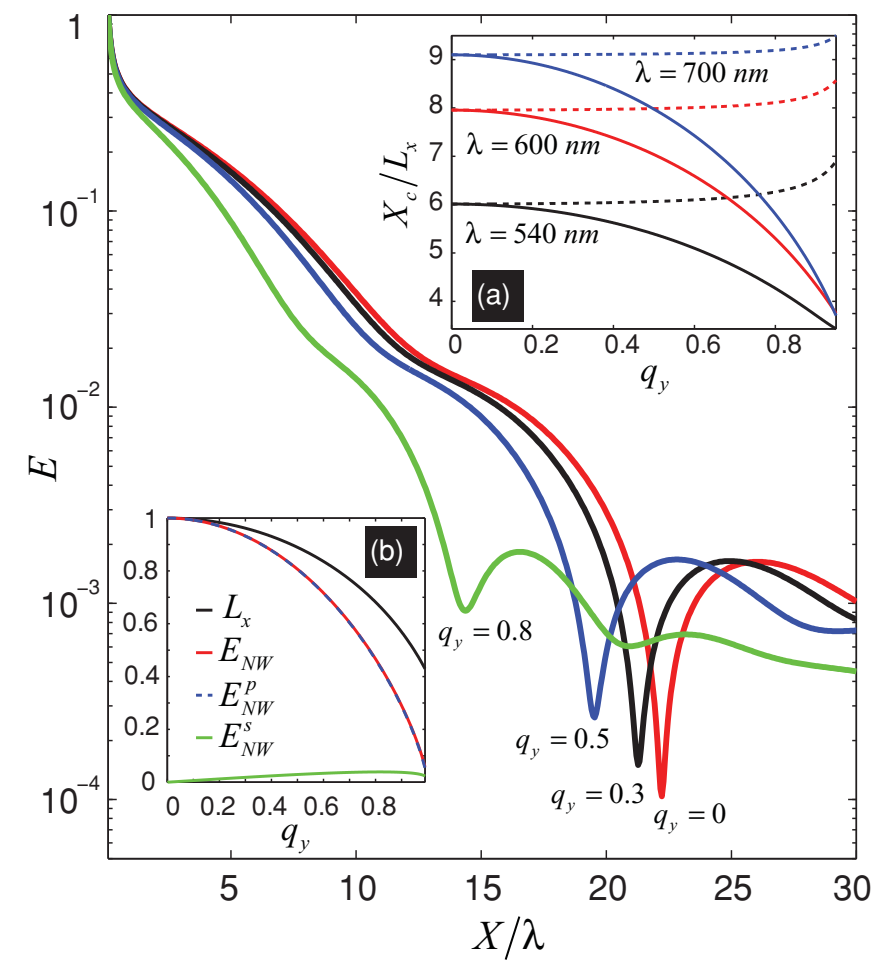

FIG. 4. (Color online) Dependency on distance from the slit of the electric field modulus, at the vacuum-gold surface for different values of $q_{y}$. The wavelength is $\lambda=540 \mathrm{~nm}$. The normalization of the fields is the same as in Fig. 3. Inset (a) shows the dependency of the ratio between the crossover distance and SPP propagation length ratio, $X_{c} / L_{x}$, as a function of $q_{y}$ and for different wavelengths. The continuous curves are for $X_{c}\left(q_{y}\right) / L_{x}\left(q_{y}=0\right)$, while the discontinuous ones are for $X_{c}\left(q_{y}\right) / L_{x}\left(q_{y}\right)$. Inset (b) renders the normalized SPP propagation length $L_{x}\left(q_{y}\right) / L_{x}\left(q_{y}=0\right)$ and the NW field modulus $E_{\mathrm{NW}}$, together with its $p$ - and $s$-polarization components, all of them normalized to $E_{\mathrm{NW}}\left(q_{y}=0\right)$.

to $q_{y}=1$. We would like to stress that while $L_{x}$ is dependent upon $q_{y}$, the SPP propagation length, $L_{\mathrm{SPP}}=\lambda /\left[2 \pi \operatorname{Im}\left(q_{p}\right)\right]=$ $L_{x}\left(q_{y}=0\right)$, showing the decrease of SPP along the direction of the Poynting vector, is not.

Inset (b) of Fig. 3 shows the NW amplitude as a function of $q_{y}$. This behavior is due to the dependence with $q_{y}$ of the spaceindependent prefactors in the amplitude of the NW (which for $\left|\varepsilon_{m}\right| \gg 1$, goes as $E_{\mathrm{NW}}^{p} \propto q_{0}^{3 / 2}$, and thus decreases as $q_{y}$ increases). By contrast, in the case of a SPP this prefactor is practically independent of $q_{y}$. However, the SPP decay length scales as $L_{x} \propto q_{0}$, so the SPP decays faster for larger values of $q_{y}$. As a result, as $q_{y}$ increases, the NW overtakes the SPP closer to the slit, but with a smaller amplitude.

As follows from Eqs.(7) and (11), the $s$-polarization component of the NW has a nonmonotonic dependency upon $q_{y}, E_{\mathrm{NW}}^{s} \propto q_{y} \sqrt{q_{0}}=q_{y} \sqrt{\varepsilon-q_{y}^{2}}$. Nonetheless, the contribution of this component is always much smaller than that from $p$ polarization [see inset (b) of Fig. 4]. Notice that, as the NW presents the same algebraic decay for all $q_{y}$ $\left(E_{\mathrm{NW}} \propto 1 / x^{3 / 2}\right)$, the normalization to $E_{\mathrm{NW}}\left(q_{y}=0\right)$ makes the quantities represented in inset (b) of Fig. 4 independent upon distance. Thus, even for oblique incidence, NWs are virtually

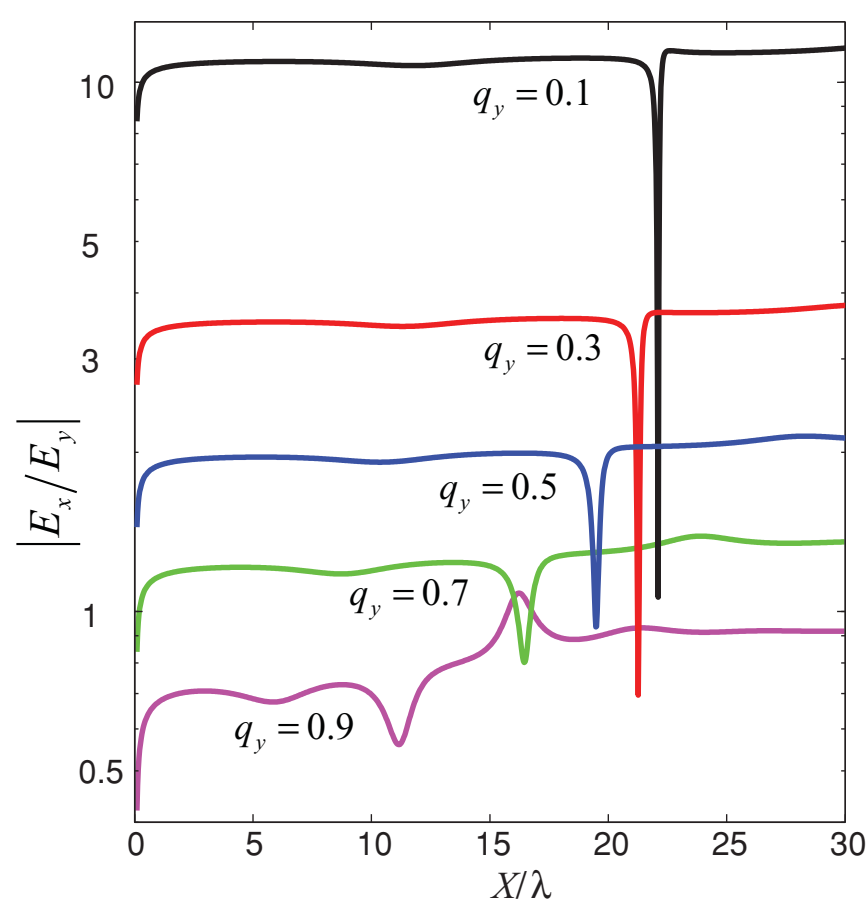

FIG. 5. (Color online) Dependency of the ratio $E_{x} / E_{y}$ upon distance from the slit for different values of $q_{y}$. The wavelength is $\lambda=540 \mathrm{~nm}$ and the considered metal is gold.

$p$-polarized waves along the interface, i.e., present the same polarization as SPPs.

It is also interesting to study the ratio $E_{x} / E_{y}$, i.e., the polarization of the tangential-to-the-interface component of the field. For the case of in-plane launching $\left(q_{y}=0\right), E_{y}=0$, and the field in the plane of the metal surface has only an $x$ component. When the excited waveguide mode gains nonzero momentum in the direction along the slit, the scattered electric field possesses a finite $y$ component in both far- and near-field regions. Figure 5 illustrates the spatial dependency of $\left|E_{x} / E_{y}\right|$ for different $q_{y}$, showing that this ratio increases with $q_{y}$. According to Eqs. (6), for a SPP the ratio $E_{x} / E_{y}$ scales as $\sqrt{\left(q_{p} / q_{y}\right)^{2}-1}$. For a NW, from Eq. (7) and neglecting the contribution from the $s$ polarization [which scales as $O\left(1 / \varepsilon_{m}\right)$ ], it follows that this ratio scales in a similar way: $E_{x} / E_{y} \simeq$ $\sqrt{1 / q_{y}^{2}-1}$. Notice that crossover distance is slightly different for $x$ and $y$ components of the electric field and, also, that the amplitudes of $E_{x}$ and $E_{y}$ are different at the crossover. For these reasons, the curves in Fig. 5 present dips at distances close to the corresponding crossovers, where both $E_{x}$ and $E_{y}$ are strongly suppressed, but $E_{y}$ dominates.

\section{CONCLUSIONS}

To conclude, in this paper we have presented the asymptotic expression for the EM field along the metal interface, launched by a subwavelength slit in the conical mount. These expressions are very accurate for the region down to a tenth of a wavelength. The field presents different contributions, which can be assigned to specific regions in the angular spectrum. The $s$-polarization component, although present for oblique incidence, does not influence significantly the behavior of the 
field. We have studied the dependencies of the fields upon the angle of incidence (i.e., the component of the EM wave vector along the slit axis). In the far-field region, the distance from the slit at which the algebraic behavior of the field overcomes the exponential decay decreases as $q_{y}$ increases. This could favor experimental studies of the Norton waves.

\section{ACKNOWLEDGMENTS}

The authors acknowledge support from the Spanish Ministry of Science and Innovation under Grant Nos. MAT200906609-C02 and CSD2007-046-NanoLight.es. A.Y.N. acknowledges the Juan de la Cierva Grant No. JCI-2008-3123.

\section{MATHEMATICAL TREATMENT OF THE FIELD AND GREEN'S DYADIC}

In this Appendix we present the details of the analytical computations for obtaining the field along the metal interface. The coordinate system is shown in Fig. 1 with $Z=0$ corresponding to the exit interface.

The general self-consistent form of the field is given by the Lippmann-Schwinger integral equation ${ }^{23}$

$$
\mathbf{E}(\mathbf{R})=\mathbf{E}_{0}(\mathbf{R})+k_{\omega}^{2} \int_{V} d \mathbf{R}^{\prime} \Delta \varepsilon\left(\mathbf{R}^{\prime}\right) \hat{\mathcal{G}}\left(\mathbf{R}, \mathbf{R}^{\prime}\right) \mathbf{E}\left(\mathbf{R}^{\prime}\right),
$$

where $\mathbf{E}_{0}$ is the solution without the slit, $\Delta \varepsilon(\mathbf{R})=\varepsilon-\varepsilon_{m}$ is the variation of the dielectric permittivity in the volume occupied by the slit $V$ ( $\Delta \varepsilon=0$ outside the slit). The film is supposed to be optically thick, so that $\mathbf{E}_{0}=0$ in the region of transmission, and the Green's function dyadic $\hat{\mathcal{G}}$ can be approximated by the one for the metal-vacuum interface, which satisfies the equation

$$
\nabla \times \nabla \times \hat{\mathcal{G}}\left(\mathbf{R}, \mathbf{R}^{\prime}\right)-k_{\omega}^{2} \epsilon \hat{\mathcal{G}}\left(\mathbf{R}, \mathbf{R}^{\prime}\right)=\hat{I} \delta\left(\mathbf{R}-\mathbf{R}^{\prime}\right),
$$

with standard boundary conditions at $z=0$. In Eq. (A2) $\hat{I}$ is a diagonal unit matrix; $\epsilon=\varepsilon$ inside the dielectric and $\epsilon=\varepsilon_{m}$ inside the metal. For convenience, let us introduce the dimensionless coordinates $x, y, z=k_{\omega} X, k_{\omega} Y, k_{\omega} Z$. Then we assume that the slit is thin enough so that the field inside it has predominantly an $x$ component and therefore only the $x$ column of $\hat{\mathcal{G}}$ will be essential, $\mathcal{G}=\mathbf{e}_{x} \hat{\mathcal{G}}$. This vector is represented by a two-dimensional integral in $k$ space (see Ref. 3), and can be decomposed in $p$ - and $s$-polarization contributions $\mathcal{G}=\mathcal{G}^{p}+\mathcal{G}^{s}$, where

$$
\mathcal{G}^{s, p}\left(\mathbf{r}, \mathbf{r}^{\prime}\right)=\frac{i k_{\omega}}{8 \pi^{2}} \int d q_{x} d q_{y} \mathbf{a}^{s, p} e^{i\left(\mathbf{q} \boldsymbol{\rho}-q_{z m} z^{\prime}+q_{z} z\right)},
$$

$\mathbf{q}=\left(q_{x}, q_{y}\right) \quad$ with $\quad q_{x, y}=k_{x, y} / k_{\omega}, \quad q_{z}=\sqrt{\varepsilon-q^{2}}, \quad q_{z m}=$ $\sqrt{\varepsilon_{m}-q^{2}}$, and $\boldsymbol{\rho}=\left(x-x^{\prime}, y-y^{\prime}\right)$. The vectors $\mathbf{a}^{s}, \mathbf{a}^{p}$ are defined to be

$$
\mathbf{a}^{p}=\frac{t^{p}}{q^{2} \sqrt{\varepsilon_{m} \varepsilon}}\left(\begin{array}{c}
q_{x}^{2} q_{z} \\
q_{x} q_{y} q_{z} \\
-q_{x} q^{2}
\end{array}\right), \quad \mathbf{a}^{s}=\frac{t^{s}}{q^{2} q_{z m}}\left(\begin{array}{c}
q_{y}^{2} \\
-q_{x} q_{y} \\
0
\end{array}\right),
$$

with $t^{p}, t^{s}$ being the Fresnel coefficients given by Eq. (3). Taking into account that the field inside the slit is given by the fundamental mode and taking into account the momentum conservation along $y$, we can write $\mathbf{E}\left(x^{\prime}, y^{\prime}, z^{\prime}\right)=\mathbf{e}_{x} E\left(x^{\prime}, z^{\prime}\right) e^{i q_{y 0} y^{\prime}}$, where $q_{y 0}$ is the dimensionless $y$ component of the incidentwave wave vector. Extracting the $y$ dependency of Green's dyadic, we have the integral

$$
\int_{-\infty}^{\infty} d y^{\prime} e^{i\left(q_{y 0}-q_{y}\right) y^{\prime}+i q_{y} y}=2 \pi \delta\left(q_{y 0}-q_{y}\right) e^{i q_{y 0} y} .
$$

Then the integration in Eq. (A3) in $q_{y}$ is performed trivially. From Eq. (A3) it follows that the integrand contains the exponential factor $e^{-i q_{z m} z^{\prime}}$, which decays at the distance of a skin depth $\delta=1 / \operatorname{Im}\left(q_{z m}\right)$, and is of the order of a few tens of $\mathrm{nm}$ in the optical regime. We can, therefore, extend the integration limits in $z^{\prime}$ to $[-\infty, 0]$. Additionally, the variation of the dyadic is much faster than that of the field inside the slit, hence the electric field inside the slit can be approximated by its value at the distance $z=-\delta$ (the average distance to the surface, weighted by the exponential decay of the field)

$$
\int_{-h}^{0} d z^{\prime} e^{-i q_{z m} z^{\prime}} \simeq \int_{-\infty}^{0} d z^{\prime} e^{-i q_{z m} z^{\prime}}=\frac{i}{q_{z m}} \simeq \frac{i}{\sqrt{\varepsilon_{m}-\varepsilon}} .
$$

Then Eq. (A1) becomes

$$
\mathbf{E}(x, y, z)=C \int_{L} d x^{\prime} \mathbf{G}\left(x-x^{\prime}, y, z\right) E_{x}\left(x^{\prime}, z^{\prime}=-\delta\right),
$$

where $C=i \sqrt{\varepsilon_{m}-\varepsilon} / k_{\omega}$ and $\mathbf{G}=\mathbf{G}^{p}+\mathbf{G}^{s}$ with

$$
\mathbf{G}^{s, p}\left(x-x^{\prime}, y, z\right)=\frac{i k_{\omega}}{4 \pi} \int d q_{x} \mathbf{a}^{s, p} e^{i\left[q_{x}\left(x-x^{\prime}\right)+q_{y} y+q_{z} z\right]} .
$$

For brevity we have omitted " 0 " in $q_{y 0}$. We have thus recovered Eqs. (1)-(3).

Taking into account the presence of the poles (placed at $q_{z} \varepsilon_{m}+q_{z m} \varepsilon=0$ ) and branch cuts and branch points [defined by $\operatorname{Im}\left(q_{z}\right)=0$ ], an asymptotic analysis of the integral (A8) can be made following the general recipes described in Ref. 20, as was done for the case $q_{y}=0$ in Ref. 11. Concentrating on the field at the interface $z=0$, we simplify $\mathbf{G}$ in the following way:

$$
\mathbf{G}(x, z=0) \equiv \frac{i k_{\omega}}{2 \pi} \mathbf{g}(x) .
$$

The branch cuts $\operatorname{Im}\left(q_{z}\right)=0$ can be removed by changing to polar variables: $q_{z}=q_{0} \cos \phi$, or $q_{x}=q_{0} \sin \phi$ with $q_{0}^{2}=$ $\varepsilon-q_{y}^{2}$. Here we assume that the contribution of the branch cuts $\operatorname{Im}\left(q_{z m}\right)=0$ is negligible, being of order $\sim e^{-\left|\sqrt{\varepsilon_{m}}\right| x}$ (otherwise, some modifications in the solution scheme would be necessary). Then, to provide the exponential decay of the integrand, the variable $\phi$ is further transformed into the variable $s$ as follows: $\sin \phi=1+i s^{2}$, so that the saddle point is placed at $s=0$. With this change the vector $\mathbf{g}$ reads

$$
\begin{gathered}
\mathbf{g}(x)=e^{i q_{0} x} \int_{L} d s \boldsymbol{\Phi}(s) e^{-q_{0} x s^{2}}, \\
\boldsymbol{\Phi}(s)=\frac{d \phi}{d s} \cdot \frac{\mathbf{a}\left[q_{x}(s)\right]}{2} q_{0} \cos [\phi(s)],
\end{gathered}
$$

where the integration path $L$ corresponds to the real axis in the complex plane $q$. Then the singular part of the integrand is separated

$\boldsymbol{\Phi}(s)=\frac{\mathbf{C}_{p}}{s-s_{p}}+\boldsymbol{\Phi}_{0}(s), \quad \boldsymbol{\Phi}_{0}(s)=\frac{\boldsymbol{\Phi}(s)\left(s-s_{p}\right)-\mathbf{C}_{p}}{s-s_{p}}$, 
where $s_{p}=e^{-i \frac{\pi}{4}} \sqrt{q_{x p} / q_{0}-1}, q_{x p}$ being the $x$ component of the SPP wave vector, which satisfies $q_{p}^{2}=q_{x p}^{2}+q_{y}^{2}=$ $\varepsilon \varepsilon_{m} /\left(\varepsilon+\varepsilon_{m}\right)$. We would like to remind the reader that only $p$-polarization type of poles are physical [i.e., they satisfy the radiation condition $\operatorname{Im}\left(q_{z}\right)>0$ ], and therefore the separation (A11) has sense only for the $p$-polarization component of $\boldsymbol{\Phi}$. The elements of the vector $\mathbf{C}_{p}$ are given by the residues of the integrand defined by Eq. (6).

Transforming the integration contour $L$ to the real axis in the complex plane $s$, the singular part of $\mathbf{g}$ can be represented using the complementary error function erfc

$$
\begin{gathered}
\mathbf{g}(x)=i \pi \mathbf{C}_{p} e^{q_{0} x\left(i-s_{p}^{2}\right)} \operatorname{erfc}\left(-i s_{p} \sqrt{q_{0} x}\right)+\mathbf{g}_{0}(x), \\
\mathbf{g}_{0}(x)=e^{i q_{0} x} \int_{-\infty}^{\infty} d s e^{-q_{0} x s^{2}} \boldsymbol{\Phi}_{0}(s) .
\end{gathered}
$$

The integral appearing in the nonsingular term of $\mathbf{g}$ can be represented in the form of an infinite sum resulting from the integration of the Tailor expansion for $\boldsymbol{\Phi}_{0}(s)$. This series reads

$$
\mathbf{g}_{0}(x)=\left.e^{i q_{0} x} \sum_{n \in \text { even }} \frac{1}{n !} \frac{d^{n} \boldsymbol{\Phi}_{0}(s)}{d s^{n}}\right|_{s=0} \frac{\Gamma\left(\frac{1+n}{2}\right)}{\left(q_{0} x\right)^{\frac{1+n}{2}}},
$$

where $\Gamma$ is a gamma function. For a large range of $x$, only the two first terms of this expansion are important. With these two terms in Eq. (A13), i.e., with the precision up to $O\left(x^{5 / 2}\right)$, Eq. (A12) transforms to Eqs. (5)-(7).

In the far-field region, which formally occurs for $\left|s_{p}\right| \sqrt{q_{0} x} \gg 1$ the asymptotic expansion of the complementary error function can be used:

$\operatorname{erfc}\left(-i s_{p} \sqrt{q_{0} x}\right)=2+\frac{e^{s_{p}^{2} q_{0} x}}{s_{p} \sqrt{\pi q_{0} x}} \sum_{n=0}^{\infty} \frac{(-1)^{n}}{(-i)^{2 n+1}} \frac{(2 n) !}{n !\left(4 s_{p}^{2} q_{0} x\right)^{n}}$,

where we have taken into account that $\operatorname{Im}\left(s_{p}\right)<0$. Formally, this asymptotic expansion is valid for distances such that $\left|s_{p}\right| \sqrt{q_{0} x} \gg 1$. However, comparisons with the exact results for the fields (obtained from the numerical computation of relevant integrals) show that usually this condition is too restrictive and the asymptotic expansion is valid even for shorter distances. *alexeynik@rambler.ru

†'lmm@unizar.es

${ }^{1}$ W. L. Barnes, A. Dereux, and T. W. Ebbesen, Nature (London) 424, 824 (2003).

${ }^{2}$ A. V. Zayats, I. I. Smolyaninovb, and A. A. Maradudin, Phys. Rep. 408, 131 (2005).

${ }^{3}$ L. Novotny and B. Hetch, Principles of Nano-Optics (Cambridge University, New York, 2006).

${ }^{4}$ S. A. Maier, Plasmonics: Fundamentals and Applications (Springer-Verlag, New York, 2006).

${ }^{5}$ P. Lalanne and J. P. Hugonin, Nat. Phys. 2, 551 (2006).

${ }^{6}$ F. López-Tejeira, S. R. Rodrigo, L. Martín-Moreno, F. J. GarcíaVidal, E. Devaux, T. W. Ebbesen, J. R. Krenn, I. P. Radko, S. I. Bozhevolnyi, M. U. González, J. C. Weeber, and A. Dereux, Nat. Phys. 3, 324 (2007).

${ }^{7}$ M. U. González, A. L. Stepanov, J.-C. Weeber, A. Hohenau, A. Dereux, R. Quidant, and J. R. Krenn, Opt. Lett. 32, 2704 (2007). ${ }^{8}$ S. Randhawa, M. U. González, J. Renger, S. Enoch, and R. Quidant, Opt. Express 18, 14496 (2010).

${ }^{9}$ B. Ung and Y. Sheng, Opt. Express 16, 9073 (2008).

${ }^{10}$ W. Dai and C. M. Soukoulis, Phys. Rev. B 80, 155407 (2009).

${ }^{11}$ A. Yu. Nikitin, S. G. Rodrigo, F. J. García-Vidal, and L. MartínMoreno, New J. Phys. 11, 123020 (2009).
${ }^{12}$ A. Yu. Nikitin, F. J. García-Vidal, and L. Martín-Moreno, Phys. Status Solidi RRL 4, 250 (2010).

${ }^{13}$ P. Lalanne, J. P. Hugonin, H. T. Liu, and B. Wang, Surf. Sci. Rep. 64, 453 (2009).

${ }^{14}$ F. López-Tejeira, F. J. García-Vidal, and L. Martín-Moreno, Phys. Rev. B 72, 161405 (2005).

${ }^{15}$ A. Drezet, A. L. Stepanov, A. Hohenau, B. Steinberger, N. Galler, H. Ditlbacher, A. Leitner, F. R. Aussenegg, J. R. Krenn, M. U. Gonzalez, and J.-C. Weeber, Europhys. Lett. 74, 693 (2006).

${ }^{16}$ R. Gordon, Phys. Rev. B 74, 153417 (2006).

${ }^{17}$ A. Yu. Nikitin and L. Martín-Moreno, Phys. Rev. B 75, 081405(R) (2007).

${ }^{18}$ M. Kuttge, H. Kurz, J. Gómez Rivas, J. A. Sánchez-Gil, and P. Haring Bolívar, J. Appl. Phys. 101, 023707 (2007).

${ }^{19}$ A. Yu. Nikitin, F. J. García-Vidal, and L. Martín-Moreno, Phys. Rev. Lett. 105, 073902 (2010).

${ }^{20}$ L. P. Felsen and N. Marcuvitz, Radiation and Scattering of Waves (IEEE, Piscataway, NJ, 1994).

${ }^{21}$ Handbook of Optical Constants of Solids, edited by E. D. Palik (Academic, New York, 1985).

${ }^{22}$ K. A. Norton, Proc. IRE 24, 1367 (1936).

${ }^{23}$ O. J. F. Martin, C. Girard, and A. Dereux, Phys. Rev. Lett. 74, 526 (1995). 